\title{
MOTORES DE COMBUSTIÓN INTERNA (MCI) OPERANDO CON MEZCLAS DE ETANOL GASOLINA: REVISIÓN
}

\author{
INTERNAL COMBUSTION ENGINES (ICE) FUELLED USING ETHANOL- \\ GASOLINE BLENDS: REVIEW
}

Alexander García Mariaca', Edwin Darío Cendales Ladino², Andrés Felipe Eslava Sarmiento³

Fecha de recepción: 19 de diciembre de 2014

Fecha de aprobación: 5 de enero de 2016

Referencia: A. García Mariaca, E. D. Cendales Ladino, A. F. Eslava Sarmiento (2016). Motores de combustión interna (MCI) operando con mezclas de etanol gasolina: revisión. Ciencia e Ingeniería Neogranadina, 26 (1), pp. 75-96, D0I: http://dx.doi.org/10.18359/rcin.1626

\section{RESUMEN}

En este artículo se presenta una revisión bibliográfica acerca del uso de etanol como combustible en motores de combustión interna de encendido provocado ( $\mathrm{MCl}-\mathrm{EP})$. Este empieza por una breve descripción de las diversas formas de producción y principales productores en el mundo de etanol, para posteriormente profundizar en el desempeño, combustión y las emisiones en MClEP al operar con diferentes mezclas de etanol y gasolina convencional. Los resultados obtenidos por los diferentes autores de los parámetros de desempeño, combustión y emisiones, como potencia, torque, presión media efectiva al freno (BME), eficiencia térmica, tasa de liberación de calor, eficiencia de combustión, presión en la cámara de combustión y emisiones reguladas ( $N O x, T H C, C O, \mathrm{CO}_{2}$ y MP), muestran que el etanol es un excelente comburente para utilizarse en mezcla con gasolina, especialmente en relaciones superiores al $20 \%$ en volumen (E20); debido a que se produce un mejor proceso de combustión causado por el aumento en la cantidad de oxígeno, y se logran así mejoras en los parámetros de desempeño y emisiones del MCl, tales como incrementos en la potencia y la eficiencia térmica y disminución de las emisiones de CO y THC. Sin embargo, se generan aumentos en algunas emisiones contaminantes, como el $\mathrm{CO}_{2}$ y los

\footnotetext{
1. Ing. Mecánico, Profesor de tiempo completo, Fundación Universitaria Los Libertadores, Bogotá, Colombia, Programa de Ingeniería Mecánica, agarciam04@libertadores.edu.co

2. Ing. Mecánico, M.Sc., Profesor de tiempo completo, Fundación Universitaria Los Libertadores, Bogotá, Colombia, Programa de Ingeniería Mecánica edcendalesl@libertadores.edu.co

3. Ing. Mecánico, Profesor de tiempo completo, Fundación Universitaria Los Libertadores, Bogotá, Colombia, Programa de Ingeniería Mecánica, afeslavas@libertadores.edu.co
} 
NOx causados por el exceso de oxígeno en la combustión y el incremento de la temperatura de los gases de escape.

Palabras clave: etanol, MCl, emisiones, desempeño, combustión.

\section{ABSTRACT}

This article presents a literature review about of the use of ethanol as fuel in internal combustion engines spark ignition (ICE-SI). This begins with a brief description of the various forms of production and major global producers of ethanol, to further deepen in the performance, combustion and regulated emissions the ICE-SI operating with different blends of ethanol with conventional gasoline. The results obtained by different authors on performance, combustion and emissions parameters, such as power, torque, brake mean effective pressure, thermal efficiency, heat release rate, efficiency of combustion pressure in the combustion chamber and regulated emissions ( $\mathrm{NOx}, \mathrm{HC}, \mathrm{CO}, \mathrm{CO}_{2}$ and $\mathrm{PM}$ ), show that ethanol is an excellent fuel to be used in blends with conventional gasoline, especially in higher ratios in volume than $20 \%$ by volume (E20); because is produced a better combustion process caused by the increase in the amount of oxygen, thus achieving improvements in performance parameters and emissions in the ICE$\mathrm{SI}$ such as increases in power and thermal efficiency and reduced emissions of CO and THC. However, are generated increases in some polluting emissions such as $\mathrm{CO}_{2}$ and NOx caused by the excess oxygen in the combustion and increasing the temperature of the exhaust gases the engines.

Keywords: ethanol, ICE, emissions, performance, combustion.

\section{INTRODUCCIÓN}

En la actualidad se presentan dos amenazas ambientales debido al alto consumo de combustibles fósiles utilizados para satisfacer la demanda energética mundial [1], la primera de estas problemáticas es la deforestación de los bosques y selvas causada por las actividades de extracción del hidrocarburos, y la segunda es provocada por los gases de efecto invernadero emitidos en los procesos de combustión de los combustibles derivados del petróleo. [1]
Por otro lado, la crisis del petróleo en la década del setenta y la continua fluctuación del precio del crudo debido a factores económicos y políticos, incentivó a los países que presentan poca disponibilidad de este recurso a buscar fuentes de energías alternativas que pudieran reducir la dependencia que se tiene de los combustibles fósiles [2]. Uno de los principales dispositivos donde son utilizados los combustibles derivados del petróleo son los motores de combustión interna (MCl), que son principalmente usados en el sector transporte; razón por la cual en las grandes ciudades las 
emisiones reguladas ( $\mathrm{NO}, \mathrm{HC}, \mathrm{CO}, \mathrm{CO}_{2}$ y $\mathrm{PM}$ ) superan los límites permitidos [3].

Estas emisiones contaminantes están causando graves problemas de salud, principalmente respiratorios a los habitantes de las grandes ciudades, con lo que se desmejora la calidad de vida y, por otro lado, se genera un incremento en los costos de la salud pública [4]. Es por eso que varias tendencias en la investigación se enfocan en el desarrollo de combustibles alternativos que sean de carácter renovable, los cuales puedan reducir o suplir la dependencia que se tiene de los combustibles fósiles [5].

Muchas de estas investigaciones exploran diversas fuentes de energía renovable, donde los más comunes son: el gas natural, propano, etanol, metanol, hidrógeno y los biocombustibles de primera, segunda y tercera generación [6]. Sin embargo, ninguna de estas fuentes alternativas es una solución total al problema que está generando el uso de combustibles fósiles. No obstante, sí presentan una gran ventaja, debido a que el uso de este tipo de combustibles disminuye las emisiones de efecto invernadero y, además, la producción de muchos de estos combustibles no causa daño a los diversos ecosistemas del planeta [7].

Los biocombustibles líquidos presentan las mejores propiedades para su uso en $\mathrm{MCl}$, esto debido a que sus propiedades fisicoquímicas son similares a los combustibles fósiles. Por otro lado, la materia prima para su producción está ampliamente disponible, lo cual no incrementa los costos de elaboración de los biocombustibles [8]. Estos se encuentran clasificados en tres categorías, los de primera generación (1G), segunda generación (2G) y tercera generación (3G). Los $1 \mathrm{G}$ son producidos a partir de cultivos alimenticios, como maíz, caña de azúcar, remolacha, etc., lo cual disminuye su viabilidad para la producción de biocombustibles. Los $2 \mathrm{G}$ son provenientes de biomasa residual o aceites vegetales tóxicos, como la Jatropha. Son los más indicados para la producción de combustibles, ya que no provienen de cultivos alimenticios. Por último, están los 3G, los cuales son producidos a partir de algas, estos presentan las mejores características para la producción de biocombustibles líquidos. Sin embargo, aún se encuentran en etapa experimental, lo cual hace que su obtención sea aún costosa [1].

El etanol, por pertenecer a los biocombustibles $1 \mathrm{G}$ y $2 \mathrm{G}$, presenta las mejores proyecciones para convertirse en el biocombustible de mayor uso en el mundo, ya que puede ser utilizado de manera directa o mezclado con combustibles de origen fósil en $\mathrm{MCl}$ de encendido provocado (EP) o en $\mathrm{MCl}$ encendidos por compresión (EC) [9].

\section{ETANOL ANHIDRO E HIDRATADO}

El etanol es un combustible alternativo perteneciente a los biocombustibles de primera y segunda generación [10], el cual puede ser utilizado en $\mathrm{MCl}$ de forma directa o mezclado con gasolina o diésel. Este proviene de cultivos como caña, papa, remolacha (biomasa con alto contenido de azúcar o almidón) o biomasa lignocelulosa [11]. Las principales formas de producción de etanol son: [11]

- Por fermentación de azúcar derivado de almidón de cereales (trigo y maíz), remolacha o cultivos de azúcar que usan microorganismos.

- Utilización de etanol excedente del vino; fermentación de las fracciones de 
lignocelulosa no azucarada de los cultivos (pastos y árboles).

- Por obtención sintética, a través de la hidratación directa de etileno (derivado del petróleo).

- Por conversión catalítica a alta temperatura de gas de síntesis para obtener combustibles líquidos por medio del proceso de Fischer-Tropsch, producción de una mezcla de alcoholes.

De los métodos mencionados, el más utilizado es la fermentación. Este se realiza por medio de microorganismos y enzimas, como levaduras, que en ausencia de oxígeno convierten los carbohidratos contenidos en la materia prima en etanol [12]. En la Tabla 1 se muestran algunas propiedades físico-químicas del etanol.

Tabla 1. Propiedades físico-químicas del etanol

\begin{tabular}{|l|c|}
\hline Propiedades del combustible & Etanol \\
\hline Número de octano & 108 \\
\hline Temperatura de autoignición [K] & 606 \\
\hline Calor latente de vaporización [MJ/kg] & 0,91 \\
\hline Poder calorífico inferior [MJ/kg] & 26,7 \\
\hline
\end{tabular}

Fuente: Balat, 2009 [13] y Kumar, 2010. [14]

Otro proceso interesante para la producción de etanol por su bajo costo, es obteniéndolo a partir de gas de síntesis. El gas de síntesis puede producirse a partir de gas natural reformado, gasificación del carbón o gasificación de biomasa residual $[15,16]$. Adicionalmente, el gas de síntesis obtenido a partir de biomasa presenta ventajas con respecto a otras formas de producción, de las cuales se resalta la gran disponibilidad de la materia prima, ya que permite el uso de una gama muy amplia de biomasa para transformar termoquímicamente mediante la gasificación [17]; convirtiendo así al etanol en un biocombustible $2 \mathrm{G}$. Este tipo de producción puede tener diversas aplicaciones; una de estas puede ser en zonas no interconectadas (ZNI), lo que facilita la producción de energía en regiones con difícil acceso y con carencia de servicios públicos [5].

La principal ventaja que tiene la utilización de etanol en $\mathrm{MCl}$, es que estos no requieren de grandes modificaciones en su diseño para que funcionen adecuadamente [1]. Además, las emisiones producidas por este biocombustible son menores que si se utilizara gasolina o diésel [18]. Por otro lado, existe una gran producción de este biocombustible en diferentes partes del mundo, siendo los principales productores Brasil y Estados Unidos. En la Figura 1 se pueden observar las proporciones de producción mundial de etanol para el año 2006.

En Colombiael Gobierno Nacional ha fomentado el uso de biocombustibles (biodiésel de palma y etanol de caña), por medio de la Ley 693 de septiembre 19 de 2001 y reglamentó la mezcla de $10 \%$ de etanol con $90 \%$ gasolina en volumen, por medio de la Resolución 180687 de 2003, razón por la cual Colombia produce 1'200.000 litros diarios [19] para satisfacer la demanda nacional [20].

\section{DESEMPEÑO EN MCI-EP AL OPERAR CON DIFERENTES MEZCLAS DE ETANOL}

El desempeño de $\mathrm{MCl}$ se ve directamente afectado por el tipo de combustible con el que este opera. Los principales parámetros que se analizan son: el torque de salida, el consumo específico de combustible (BSFC), la eficiencia térmica al freno y la eficiencia volumétrica. 


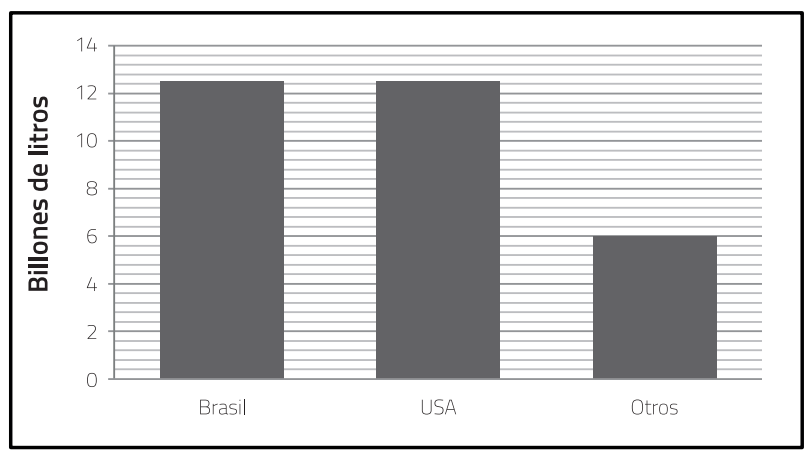

Figura 1. Producción mundial de etanol.

Fuente: Agarwal, 2007 [1]

A continuación se presentan los resultados de desempeño obtenidos por diferentes investigadores, y en la Tabla 2 un resumen de los resultados.

Costa y Sodré [21] investigaron etanol hidratado como único comburente y una mezcla E22 en un MCl-EP con una cilindrada de 1.000 $\mathrm{cm}^{3}$, de inyección directa, refrigerado por agua, variando la relación de compresión de 10:1 a 12:1 para diferentes rpm. Encontraron que el torque, BMEP y la potencia neta de salida son mayores al utilizar la relación de compresión de 12:1, para ambos combustibles especialmente a altas velocidades. Adicionalmente, los resultados obtenidos con el etanol hidratado fueron superiores a la mezcla E22 en un 1,6\% para el torque y un 3,1\% en potencia. El BSFC disminuye con el aumento de la relación de compresión para ambos combustibles, siendo mayor la disminución para el etanol hidratado. La eficiencia térmica fue mayor con el etanol hidratado a una relación de compresión de 12:1. Sin embargo, la eficiencia volumétrica presenta mejores resultados con relaciones de compresión bajas para ambos combustibles.

Hüseyin et al. [22] investigaron los parámetros de desempeño en un $\mathrm{MCl}$-EP monocilíndrico de inyección indirecta, de marca Hydra, al operar con mezclas E0, E10, E20, E40 y E60; en estas pruebas se varió la relación de compresión desde 8:1 a 13:1 y se encontró que con el aumento de la relación de compresión hasta 11:1, el torque del motor aumenta un $8 \%$ con el combustible EO a 2.000 rpm, en comparación con la relación de compresión de 8:1. Para relaciones de compresión más altas, el torque de salida no cambió notablemente. Para mezclas E40 y E60 con una relación de compresión de $13: 1$, se tuvo un $14 \%$ más de torque en comparación con la relación de compresión de 8:1. Por otro lado, el BSFC tiene una disminución del $15 \%$ con la mezcla E40 a 2.000 rpm, y con E60 a 3.000 y 5.000 rpm se obtuvo una disminución del 14,5 y $17 \%$, respectivamente.

Rong-Hong et al. [23] probaron mezclas de etanol gasolina E0, E5, E10, E20 y E30 en un MCl-EP marca New Sentra GA16DE con cilindrada de $1.600 \mathrm{~cm}^{3}$, con inyección multipunto y relación de compresión de 9,5:1. Encontraron que el torque de EO es ligeramente inferior al obtenido con las otras mezclas, especialmente para aberturas de mariposa bajas $<20 \%$ o altas velocidades del motor $>$ 4.000 rpm. Por otro lado, el BSFC permanece 
Tabla 2. Resultados de desempeño en MCl-EP al operar con diferentes mezclas de etanol-gasolina comparados con gasolina

\begin{tabular}{|c|c|c|c|c|c|}
\hline \multirow[b]{2}{*}{ Mezclas } & \multicolumn{4}{|c|}{ Comparación respecto a la gasolina [\%] } & \multirow[b]{2}{*}{ Ref. } \\
\hline & BSFC & Torque & Potencia & Eficiencia térmica & \\
\hline E22 & $2.7 \downarrow$ & $1,6 \uparrow$ & $3,1 \uparrow$ & $2,5 \uparrow$ & {$[21]$} \\
\hline E40 y E60 & $15 \downarrow$ & $14 \uparrow$ & & & {$[22]$} \\
\hline $\begin{array}{l}\mathrm{E} 5, \mathrm{E} 10, \mathrm{E} 20 \mathrm{y} \\
\mathrm{E} 30\end{array}$ & $\begin{array}{c}\text { Casi constante a bajas } \\
\text { velocidades }\end{array}$ & $\begin{array}{l}\text { Mayor para aperturas de } \\
\text { mariposa < al 20\% }\end{array}$ & & & {$[23]$} \\
\hline $\begin{array}{l}\text { E25, E50, E75 y } \\
\text { E100 }\end{array}$ & $3 \downarrow$ & & $29 \uparrow$ & & {$[24]$} \\
\hline E5 y E10 & $\begin{array}{c}3,2 \uparrow \text { a } 80 \mathrm{~km} / \mathrm{h} \\
0,85 \uparrow \text { a } 100 \mathrm{~km} / \mathrm{h}\end{array}$ & & & $\begin{array}{l}1,9 \uparrow \text { a } 80 \mathrm{~km} / \mathrm{h} \\
2,5 \uparrow a 100 \mathrm{~km} / \mathrm{h}\end{array}$ & {$[25]$} \\
\hline E22 & & & $3,7 \uparrow$ & $14,1 \uparrow$ & {$[26]$} \\
\hline E10 y E20 & $\begin{array}{c}\text { Disminuye para cargas } \\
\text { medias }\end{array}$ & & & & {$[28]$} \\
\hline E100 & $56 \uparrow$ & $3,7 \uparrow$ & & & {$[29]$} \\
\hline $\begin{array}{c}E 10, E 20, E 40 \\
y E 60 \\
\end{array}$ & & Máximo a 22 CAD & & & {$[30]$} \\
\hline $\begin{array}{l}\mathrm{E5}, \mathrm{E} 10, \mathrm{E} 20 \mathrm{y} \\
\text { E30 }\end{array}$ & & $\begin{array}{c}5,8 \uparrow \text { con mezclas E20 } \\
\text { y E30 }\end{array}$ & & & {$[31]$} \\
\hline E10, E20 & $13 \downarrow$ & & & $1,3 \uparrow$ & {$[32]$} \\
\hline $\begin{array}{c}\text { E10, E20, E40 } \\
\text { y E60 }\end{array}$ & $\begin{array}{c}\text { Incrementos para todas } \\
\text { las mezclas }\end{array}$ & & & & [33] \\
\hline $\begin{array}{l}\text { E10, E20, E30, } \\
\text { E50, E85 y E100 }\end{array}$ & & & & $\begin{array}{c}\text { Máxima entre } 24 \text { y } 34 \\
\text { CAD }\end{array}$ & {$[34]$} \\
\hline E100 & & & & $\begin{array}{c}\text { Máximo con mayor masa } \\
\text { residual en la mezcla }\end{array}$ & {$[35]$} \\
\hline E100 & $6 \downarrow$ & & & & {$[36]$} \\
\hline $\begin{array}{l}\text { E5, E10, E15 y } \\
\text { E20 }\end{array}$ & $\begin{array}{c}\text { 7,2 } \uparrow \text { para E10, 7,2 } \uparrow \text { para } \\
\text { E15 } 18.77 \text { para E20 }\end{array}$ & & & & {$[37]$} \\
\hline E25 hasta E27,5 & $5,7 \uparrow$ & & $8,3 \uparrow$ & $9 \uparrow$ & {$[7]$} \\
\hline E5 y E10 & & & $\begin{array}{l}2,2 \uparrow \operatorname{con} E 5 \\
1,1 \uparrow \operatorname{con} E 10\end{array}$ & & [8] \\
\hline
\end{tabular}

Fuente: Elaboración propia.

constante a velocidades bajas (1.000 y 2.000 rpm) con aberturas de la mariposa $>20 \%$.
Celik [24] evaluó mezclas de etanol gasolina de E0, E25, E50, E75 y E100, en MCl-EP 
marca Lombardi LM 250, de carburador, con relación de compresión de 6:1 y 10:1, a una velocidad constante de 2.000 rpm, y encontró que la mezcla ideal donde se obtiene la mayor salida de potencia y las emisiones más bajas de THC es cuando el motor opera con la mezcla E50. De los resultados experimentales, se determinó que la potencia del motor aumenta aproximadamente $29 \%$ cuando el MCI-EP opera con E50 a la mayor relación de compresión de prueba, en comparación a EO, al mismo tiempo que el BSFC se reduce un $3 \%$ aproximadamente para la misma condición de operación.

Eyidogan et al. [25] realizaron pruebas en un dinamómetro de rodillos a dos condiciones de velocidad: 80 y $100 \mathrm{~km} / \mathrm{h}$, en un motor enfriado por agua, de inyección multipunto de 1.396 $\mathrm{cm}^{3}$, con relación de compresión de 10,4:1, probaron mezclas E5 y E10, y encontraron que a $80 \mathrm{~km} / \mathrm{h}$ el BSFC para E5 y E10 aumentó un 2,8 y $3,6 \%$, respectivamente, en comparación con EO. A una velocidad de $100 \mathrm{~km} / \mathrm{h}$ el BSFC para E5 y E10 se incrementó en 0,2 y 1,5\%, respectivamente. Por otro lado, encontraron que a $100 \mathrm{~km} / \mathrm{h}$ los incrementos en las eficiencias térmicas de E5, E10 son 1,9 y $2,5 \%$, respectivamente, respecto a EO.

Costa y Sodré [26], quienes investigaron etanol hidratado y una mezcla de E22 en un $\mathrm{MCl}$-EP de $1.000 \mathrm{~cm}^{3}$, de inyección, relación de compresión 12:1 y refrigerado por agua, encontraron que a velocidades de motor por debajo de 3.250 rpm, la mezcla E22 disminuye el torque y la presión media efectiva al freno (BMEP) respecto a la situación en la que el motor utiliza etanol hidratado. Para velocidades de más de 4.000 rpm, el uso de etanol hidratado resultó en mayor torque y BMEP en relación con la mezcla de combustible E22, también se obtuvo un $3,7 \%$ más potencia con etanol hidratado en comparación con la mezcla E22 para velocidades de más de 5.000 rpm. Además, el uso de etanol hidratado produjo una eficiencia térmica más alta del MCl-EP a través de todo el rango de velocidad investigado, al alcanzar un aumento máximo de 14,1\% en comparación con el E22. El BSFC del motor al operar con etanol hidratado fue alrededor de un $54 \%$ más alto que cuando se utilizó E22.

Park et al. [27] utilizaron un MCl-EP de 494 $\mathrm{cm}^{3}$, de inyección multipunto con relación de compresión 10:1, el cual fue operado con una mezcla E85 a dos condiciones de giro: 1.500 y 2.000 rpm, respectivamente. En este estudio encontraron que para las dos velocidades mencionadas, la eficiencia térmica es mayor para la mezcla E85 que para E0, y además este parámetro aumenta en el motor cuando se tiene una mezcla pobre en combustible (lambda $>1)$, sin importar el tipo de combustible con el que opere.

Zhuang y Hong [28] probaron mezclas de gasolina y etanol con el fin de evaluar la potencia generada por el motor. Para esto utilizaron un $\mathrm{MCl}-\mathrm{EP}$, monocilíndrico, enfriado por aire, con cilindrada de $249 \mathrm{~cm}^{3}$, relación de compresión 9,8:1; las pruebas sobre el motor se llevaron a bajas y medias condiciones de carga. Encontraron que la eficiencia volumétrica y el BMEP para cargas bajas es menor que para cargas medias del motor y es independiente de las rpm del motor, el BSFC es menor para cargas medias que para cargas bajas. Sin embargo, la tendencia es que este parámetro se incremente a medida que se aumenta el porcentaje de etanol en la mezcla.

Balki et al. [29] investigaron porcentajes de mezcla de E100 y E0 en un MCl-EP monocilíndrico de $196 \mathrm{~cm}^{3}$, enfriado por aire, con relación de compresión de 8,5:1, y 
encontraron que el torque máximo se obtiene a $2.400 \mathrm{rpm}$ para los dos combustibles. En promedio, el torque máximo del $\mathrm{MCl}$ con E100 se incrementó en 3,7\%, en comparación con los valores de E0. El BSFC para E100 fue un $56 \%$ mayor que para la E0, y asimismo la eficiencia térmica al freno también fue mayor para E100 con respecto a EO.

Topgül et al. [30] analizaron el desempeño en un $\mathrm{MCl}$-EP monocilíndrico de relación de compresión variable de 5:1 a 13:1, con inyección, al operar con diferentes mezclas de etanol: E0, E10, E20, E40 y E60. Determinaron que el torque máximo para una relación de compresión de 10:1 ocurre cuando el tiempo de ignición es de 22 CAD para todas las mezclas, con lo que se obtiene mejor resultado con la mezcla E20. Además, encontraron que el retraso del tiempo de encendido no varía notablemente la temperatura de los gases de escape.

Wu et al. [31] utilizaron un MCl-EP de 4 cilindros con cilindrada de $1.600 \mathrm{~cm}^{3}$ y relación de compresión de 9,5:1, para probar mezclas E0, E5, E10, E20 y E30. Observaron que con el motor operando entre 3.000 y 4.000 rpm, al $20 \%$ de apertura de la mariposa, al utilizar E5 conduce a una mayor salida de par en condición de mezcla pobre. También encontraron que al 40 \% de apertura de la mariposa y a 4.000 rpm del motor al operar con E20 y E30, los resultados en la salida de par tienen un incremento aproximado de $5,8 \%$ con respecto a EO.

Zhang et al. [32] probaron un $\mathrm{MCl}-\mathrm{EP}$ de 4 cilindros en línea, refrigerado por agua, de inyección directa con 1,8 L de cilindrada, que utilizaba gases de escape de recirculación (EGR) con mezclas de E10 y E20. Encontraron que con el aumento de la tasa de EGR, el BSFC para todos los combustibles de prueba disminuye en alrededor de 4,9 a 6,1\%.
Observaron también que la eficiencia térmica al freno puede ser aparentemente mejor con la adición de EGR en condiciones de carga alta, debido a la pérdida de la temperatura de combustión y pérdidas de transferencia de calor. La diferencia máxima en BSFC entre E20 y EO está entre 7 y $20 \%$, y la eficiencia térmica de E20 es 1,3\% mayor que el EO. Por otro lado, el E10 muestra un comportamiento cercano de BSFC con EO.

Ozsezen y Canakci [8] realizaron pruebas en un dinamómetro de rodillos a dos condiciones de velocidad: 80 y $100 \mathrm{~km} / \mathrm{h}$ en un motor enfriado por agua, de inyección multipunto de $1.396 \mathrm{~cm}^{3}$, con relación de compresión de 10,4:1. En estas pruebas encontraron que la potencia se incrementó en el motor un 2,2 \% y $1,1 \%$ cuando este operó con mezclas E5 y E10, respectivamente, con respecto a EO.

Schifter et al. [33] utilizaron un $\mathrm{MCl}-\mathrm{EP}$ monocilíndrico de 0,5 L, de inyección, con relación de compresión de 10,5:1, para probar mezclas E0, E10, E20, E30, E40, HE10, HE20, HE30 y HE40, y etanol hidratado, y encontraron que el consumo de combustible es mayor para el etanol hidratado que para el etanol anhidro para todos los porcentajes de mezclas. Lo cual es causado por el bajo poder calorífico inferior (LHV) del etanol hidratado.

Turner et al. [34] realizaron pruebas experimentales en un $\mathrm{MCl}$-EP de 8 cilindros en $V$, de inyección directa, de aspiración natural, al operar con mezclas E0, E10, E20, E30, E50, E85 y E100. En estas pruebas encontraron que la eficiencia térmica máxima del MCl para todos los combustibles de prueba se presenta para tiempos de inyección entre 24 y 34 CAD.

Wua et. al. [35] utilizaron un $\mathrm{MCl}-\mathrm{EP}$ de inyección dual, monocilíndrico de 565,6 $\mathrm{cm}^{3}$, 
con relación de compresión de 11,5:1 operando con mezclas E100 y EO. En las pruebas encontraron que la presión media efectiva indicada (IMEP) es función de la fracción de masa quemada que queda en la cámara de combustión. Además, encontraron que si se aumentan las fracciones de EO se tienen mayores ganancias de eficiencia que con E100.

Hakan [36] validó un modelo teórico con pruebas experimentales. El motor utilizado parala validación fue un $\mathrm{MCl}$-EP monocilíndrico, enfriado por agua, con relación de compresión variable de $763 \mathrm{~cm}^{3}$, los resultados muestran una relación entre los resultados teóricos y experimentales para la potencia con solo un error del $6 \%$. También encontró que la mezcla E7,5 presenta una reducción del BSFC de 5,59 y $4,94 \%$ para relaciones de compresión de $7,75: 1$ y $8,25: 1$, respectivamente.

Kumar et. al. [37] probaron mezclas de etanol gasolina E5, E10, E15 y E20 en un MCl-EP de cuatro cilindros, refrigerado con agua, con relación de compresión 9,2:1, y encontraron que el BSFC aumenta para E10, E15 y E20 un $7,2,8$ y un $18,77 \%$, respectivamente, en comparación con EO a 2.100 rpm. La eficiencia mecánica aumenta con el aumento de etanol en la mezcla y es ligeramente mayor a $4.000 \mathrm{rpm}$ en todo el rango de carga. Además, se encontró que a carga completa la eficiencia mecánica se incrementa en $11,85 \%$ para E10, 5,5\% para E15 y $10,99 \%$ para E2O a 3.000 rpm, y aumenta un $3,36 \%$ para E10, $2,89 \%$ para E15 y $1,03 \%$ para E20 a 4.000 rpm con respecto a EO.

Al-Hasan [7] realizó pruebas en un $\mathrm{MCl}-\mathrm{EP}$ de cuatro cilindros de $1.452 \mathrm{~cm}^{3}$ y relación de compresión 9:1, operando con mezclas de etanol y gasolina desde 2,5 hasta un $27,5 \%$ con incrementos de $2,5 \%$. Los resultados de este estudio mostraron que la adición de etanol en la mezcla genera un aumento de todos los parámetros de desempeño, como la potencia, la eficiencia térmica del freno, el rendimiento y el consumo de combustible en un $8,3 \%, 9,0 \%$, $7 \%$ y $5,7 \%$, respectivamente.

Canakci et al. [38] emplearon mezclas E0, E5 y E10 en un MCl-EP enfriado por agua, de inyección multipunto, con $1.398 \mathrm{~cm}^{3}$ y relación de compresión de 10,4:1. A 80 $\mathrm{km} / \mathrm{h}$ encontraron que BSFC de E5 y E10 se incrementa en 2,8 y $3,6 \%$, respectivamente, comparado con el motor operando con EO. Del mismo modo, los aumentos en el BSFC de E5 y E10 fueron de 0,2 y $1,5 \%$ a $100 \mathrm{~km} / \mathrm{h}$, en comparación con EO.

\section{COMBUSTIÓN EN MCI-EP AL OPERAR CON DIFERENTES MEZCLAS DE ETANOL}

El análisis de los procesos de combustión es muy importante en los $\mathrm{MCl}$, ya que este indica qué tanta energía es aprovechada del combustible. Los parámetros más importantes que se establecen en este análisis son la eficiencia de combustión, la presión en la cámara de combustión y la tasa de liberación de calor, la cual está definida como la tasa a la cual la energía química del combustible es liberada por el proceso de combustión [39]. A continuación se presentan los resultados obtenidos de los parámetros de combustión en $\mathrm{MCl}$ al operar con mezclas de etanol-gasolina (ver Tabla 3).

Eyidogan et al. [25], en el MCl-EP descrito anteriormente, encontraron que para ambas velocidades del vehículo $(80 \mathrm{~km} / \mathrm{h}$ y 100 $\mathrm{km} / \mathrm{h}$ ), la presión de gas en el cilindro con el uso de EO comenzó a aumentar antes que con las mezclas de combustible, especialmente para cargas de $5 \mathrm{~kW}$ y $20 \mathrm{~kW}$; esta diferencia 
Tabla 3. Resultados de combustión en MCl-EP al operar con diferentes mezclas de etanol-gasolina comparados con gasolina

\begin{tabular}{|c|c|c|c|c|}
\hline \multirow[b]{2}{*}{ Mezclas } & \multicolumn{3}{|c|}{ Comparación respecto a la gasolina [\%] } & \multirow[b]{2}{*}{ Ref. } \\
\hline & Presión de cámara & $\begin{array}{l}\text { Tasa de liberación de } \\
\text { calor }\end{array}$ & $\begin{array}{c}\text { Eficiencia } \\
\text { de combustión }\end{array}$ & \\
\hline E5 y E10 & $\begin{array}{c}\text { Aumenta con las } \\
\text { mezclas }\end{array}$ & & & {$[25]$} \\
\hline E10 y E20 & & $\begin{array}{c}\text { Menor liberación de } \\
\text { calor }\end{array}$ & $0,29 \uparrow$ & {$[40]$} \\
\hline E6, E10, E15 y E20 & & $50 \uparrow$ & & {$[34]$} \\
\hline $\begin{array}{c}\text { E0, E10, E20, E30, E50, } \\
\text { E85 y E100 }\end{array}$ & $11 \uparrow$ & & $\begin{array}{c}\text { Mejor eficiencia de } \\
\text { combustión }\end{array}$ & {$[36]$} \\
\hline E100 & $11 \uparrow$ & & $0,32 \uparrow$ & {$[8]$} \\
\hline
\end{tabular}

Fuente: Elaboración propia.

se manifiesta de forma muy significativa. Además, la presión máxima para todos los combustibles de ensayo se produjo cerca del punto muerto superior (PMS). A los 10, 15 y 20 $\mathrm{kW}$, donde el motor funciona de manera más estable, la presión de gas del cilindro con EO es más grande que con E5 y E10.

Zhuang y Hong [28], en el montaje descrito anteriormente, encontraron que la tasa de liberación de calor con el aumento de porcentaje de etanol en la mezcla es menor respecto a EO. Por otro lado, Balki et al. [29] observaron que la eficiencia máxima de combustión con etanol es un 0,29\% mayor en comparación con los valores de EO. Los cual se debe a la molécula de oxígeno que tiene el etanol en su composición química.

Shifter et al. [40] utilizaron un MCl-EP, monocilíndrico de $0.5 \mathrm{~L}$, de inyección, relación de compresión de 10,5:1; probaron mezclas E0, E6, E10, E15 y E20, y encontraron que con el aumento del porcentaje de etanol en la mezcla se incrementa el valor de lambda casi un 8 \%. Para E15 y E20 no solo la combustión es más rápida, sino que también se tiene una mayor liberación de calor instantáneo de casi el $50 \%$. Para E10, la liberación de calor es solo ligeramente más rápida que con E0, causada por el bajo LHV del etanol.

Schifter et al. [33], en el montaje descrito anteriormente, encontraron que el contenido de agua ralentiza el proceso de combustión, pero mantiene constante la cantidad de energía producida por ciclo. Asimismo, la cantidad de trabajo obtenido es la misma, libera la misma cantidad de calor, pero de manera más eficiente.

Turner et al. [34] establecieron que al variar los tiempos de inyección se obtiene una reducción en la iniciación de la combustión debido a mayores velocidades de llama laminares con las mezclas. También obtuvieron una combustión más rápida y, por tanto, una mayor presión en el cilindro, debido a una combinación de la 
combustión avanzada y velocidades de llama más altas. Además, reportaron una mejora en la estabilidad de la combustión resultante, debido a la velocidad de llama, lo que mejora la eficiencia de combustión como resultado de una mejor evaporación.

Hakan [36], en el modelo desarrollado, muestra que la presión en la cámara de combustión del motor aumenta con el contenido de etanol en la mezcla. En la validación experimental encontró que para las dos relaciones de compresión utilizadas $(7,75$ y 8,25), aumentó la presión teórica máxima del cilindro, elevándose hasta un $11 \%$. El modelo utilizado en esta investigación también pronostica que las temperaturas máximas en los cilindros aumentan alrededor de un $2 \%$ con el aumento del porcentaje de etanol. Además, la presión del cilindro y la temperatura alcanzan sus valores máximos para la mezcla E16,5.

Ozsezen y Canakci [8] encontraron que la eficiencia de combustión para E5 y E10 aumenta 0,21 y 0,43\%, respectivamente, en comparación con EO, mientras que las eficiencias de combustión encontradas para las mezclas E5, E10 y E0 se calcularon en $98,59,98,65$ y $98,37 \%$, respectivamente, con el vehículo operando a $100 \mathrm{~km} / \mathrm{h}$. Wua et al. [35], en el montaje descrito anteriormente, encontraron que la duración de la combustión dentro de la cámara se reduce en un máximo de $5 \%$ para los biocombustibles de etanol.

\section{EMISIONES EN MCI-EP AL OPERAR CON DIFERENTES MEZCLAS DE ETANOL}

Las emisiones producidas en los $\mathrm{MCl}$ son la principal desventaja tecnológica que tienen estos dispositivos. Las emisiones producidas en $\mathrm{MCl}$ se encuentran clasificadas como reguladas y no reguladas, siendo las reguladas las de mayor estudio por los investigadores. Sin embargo, las emisiones no reguladas en la actualidad se han estudiado de manera más detallada, ya que han encontrado que estas son las más perjudiciales para la salud humana [41]. A continuación se presentan los resultados obtenidos por diferentes estudios sobre emisiones producidas en $\mathrm{MCl}-\mathrm{EP}$ al operar con diferentes mezclas de gasolina y etanol, y en la Tabla 4 se presenta un resumen de los resultados encontrados.

Costa y Sodré [26] encontraron que, con el uso de etanol hidratado, disminuyen las emisiones de $\mathrm{CO}$ y $\mathrm{HC}$ pero aumentan las emisiones de $\mathrm{CO}_{2}$ y NOx, cuando se compara con mezcla E22. Por otra parte, la temperatura de los gases de escape es menor con el etanol hidratado que para la mezcla E22. Esto se debe a que el contenido de agua en el etanol hidratado puede absorber más calor en el proceso de combustión.

Hüseyin et al. [22] reportan que las mezclas E40 y E60 tuvieron efectos importantes en la emisión de $\mathrm{CO}$ y THC, y encontraron una disminución máxima con las mezclas E40 y E60 a 2.000 rpm. En promedio, la reducción de la emisión de CO es de 11 \% y 10,8 \% con E40 y E60, respectivamente. También obtuvieron la máxima disminución de la emisión de THC de $16,45 \%$ al utilizar E60 a 5.000 rpm. Además, encontraron que la temperatura de los gases de escape disminuye con el aumento de la relación de compresión.

Chang et al. [23] encontraron que las emisiones de $\mathrm{CO}$ y $\mathrm{HC}$ se pueden reducir de $10 \%$ a $90 \%$, respectivamente, con el uso de mezclas de etanol y gasolina, mientras que los aumentos de emisión de $\mathrm{CO}_{2}$ van desde $5 \%$ hasta $25 \%$, dependiendo de las condiciones de operación 
Tabla 4. Resultados de emisiones en MCI-EP al operar con diferentes mezclas de etanol-gasolina comparados con gasolina

\begin{tabular}{|c|c|c|c|c|c|c|}
\hline \multirow{2}{*}{ Mezclas } & \multicolumn{5}{|c|}{ Comparación respecto a la gasolina } & \multirow{2}{*}{ Ref. } \\
\hline & CO & THC & NOx & $\mathrm{CO} 2$ & Temperatura & \\
\hline E40 y E60 & $11 \downarrow$ & $16.45 \downarrow$ & & & & {$[22]$} \\
\hline E5, E10, E20 y E30 & $10 \downarrow$ & $10 \downarrow$ & & $5 \uparrow$ & & {$[23]$} \\
\hline E25, E50, E75 y E100 & $53 \downarrow$ & $12 \downarrow$ & $19 \downarrow$ & $10 \downarrow$ & & {$[24,34,35]$} \\
\hline E5 y E10 & & & & & $3 \mathrm{a} 25^{\circ} \downarrow$ & [25] \\
\hline E100 & $29 \downarrow$ & $13.6 \downarrow$ & & & & [29] \\
\hline E10, E20, E40 y E60 & $21 \downarrow$ & $31.45 \downarrow$ & & & & {$[30]$} \\
\hline E10 y E20 & & $11 \downarrow$ & & & & [32] \\
\hline E100 & $42 \downarrow$ & & & & & [36] \\
\hline E25 hasta E27,5 & $30 \downarrow$ & & & $7,5 \uparrow$ & & [7] \\
\hline E5 y E10 & $11 \downarrow$ & & $7.5 \uparrow$ & $0.6 \downarrow$ & $1.3^{\circ} \downarrow$ & [8] \\
\hline E10 y E20 & $52 \downarrow$ & $19 \downarrow$ & $60 \uparrow$ & & & {$[40]$} \\
\hline E10, E20, E30 y E85 & $15 \downarrow$ & $20 \downarrow$ & $15 \downarrow$ & & & [41] \\
\hline E10 y E30 & $5,2 \downarrow$ & $14,2 \downarrow$ & $33 \downarrow$ & & & [43] \\
\hline E10, E15, E20, E25 y E30 & & $20,4 \downarrow$ & & & & [44] \\
\hline E10 & $16 \downarrow$ & & $45 \downarrow$ & & & [45] \\
\hline E5, E10 y E15 & $35 \downarrow$ & $30 \downarrow$ & $38 \downarrow$ & $6,2 \downarrow$ & & [47] \\
\hline E5, E10 & $18 \downarrow$ & & $13 \downarrow$ & $8 \downarrow$ & & {$[38]$} \\
\hline E10, E20, E50 y E85 & $46 \downarrow$ & $18 \downarrow$ & $14 \uparrow$ & & & [48] \\
\hline E0, E10 y E85 & & $20 \downarrow$ & $33 \uparrow$ & & & [50] \\
\hline
\end{tabular}

Fuente: Elaboración propia.

del $\mathrm{MCl}$ de prueba. También observaron que la emisión de NOx está estrechamente relacionada con la relación de equivalencia, de tal manera que la emisión de NOx alcanza un máximo cerca de la condición estequiométrica. Celik [24] encontró que las emisiones de CO, $\mathrm{CO}_{2}, \mathrm{HC}$ y $\mathrm{NOx}$ se reducen aproximadamente un $53 \%, 10 \%, 12 \%$ y $19 \%$, respectivamente, cuando el motor es operado con E50 y con una relación de compresión de 10:1. Por su parte, Turner et al. [34] obtuvieron resultados similares de reducción de emisiones de NOx, mientras que Wua et al. [35] encontraron que los $\mathrm{HC}, \mathrm{NOx}$ y $\mathrm{CO}_{2}$ se reducen en su mayoría bajo la estrategia de inyección dual.

Ozsezen y Canakci [8] examinaron los valores medios de emisiones en todas las velocidades 
del vehículo, y encontraron que la emisión de HC disminuyó en un 8 y $14 \%$ con el uso de E5 y E10; mientras que las emisiones de CO con el uso de E5 y E10 disminuyeron en 3,4 y 2,6\%, respectivamente, a $80 \mathrm{~km} / \mathrm{h}$ y $100 \mathrm{~km} / \mathrm{h}$ en comparación con EO. En este estudio las emisiones de $\mathrm{CO}_{2}$ con el uso de $\mathrm{E} 5$ se redujeron en $0,6 \%$, mientras que las emisiones de $\mathrm{CO}_{2}$ con el uso de E10 aumentaron en un 0,1\%. Las emisiones de NOx con el uso de E10 se redujeron en $1,3 \%$, mientras que aumentaron en un $1 \%$ con el uso de E5. Además, se calculó un aumento máximo en la emisión de NOx con E5 hasta del $15 \%$ a $40 \mathrm{~km} / \mathrm{h}$. Por otro lado, las relaciones de equivalencia de todos los combustibles de ensayo a $100 \mathrm{~km} / \mathrm{h}$, velocidad del vehículo, están cerca de la relación estequiométrica; este resultado provocó un aumento de las emisiones de $\mathrm{CO}_{2}$ para todos los combustibles de ensayo.

Zhuang y Hong [28] encontraron que el BSCO para baja carga del $\mathrm{MCl}$ es menor hasta una mezcla E48; para mayores porcentajes de mezcla de etanol en gasolina, el BSCO tiene valores superiores en comparación con los reportados con el $\mathrm{MCl}$ operando a carga media. Similar comportamiento presentan las emisiones de BSHC y BSNO para las dos condiciones de carga del motor. Sin embargo, estas muestran incrementos cuando el motor opera con mezclas con un porcentaje superior a E24.

Balki et al. [29] encontraron que el uso de etanol disminuyó la emisión de HC en 13,6 \%, y las emisiones de CO se redujeron 29,07\%, en comparación con el estudio en el que se utilizó gasolina convencional. En este estudio también se puede deducir que con el aumento de la velocidad rotacional, las emisiones de CO y $\mathrm{HC}$ disminuyen.

Topgül et al. [30] determinaron que el motor al operar con la mezcla E40 obtuvo los mejores resultados respectoa la reducción deemisiones; esta reducción fue aproximadamente 31,8\% para una relación de compresión de 9:1. La mezcla E60 causó una disminución en las emisiones de CO entre 19,8 \% y 22,3\% para relaciones de compresión de 8:1 y 9:1. Por otro lado, con la mezcla E60, también se obtuvo una reducción de 31,45\% en las emisiones de HC, para una relación de compresión de 10:1.

Schifter et al. [40], en el montaje descrito anteriormente, establecieron que la disminución en las emisiones del $\mathrm{MCl}$ operando con E2O fue de $52 \%$ y $19 \%$ para el CO y $\mathrm{HC}$, respectivamente. También se obtuvo un aumento del 60 \% de la emisión de NOx para el combustible E20. Para el intervalo de valores de lambda considerado en el trabajo experimental, la mezcla E20 mostró una reducción media del $13,6 \%$ en el contenido de CO y un aumento en los HC y los NOx de alrededor de $5 \%$. Con la mezcla E15 se presenta la misma tendencia, con reducciones del $7,4 \%$ en $\mathrm{CO}$, con la mezcla E10. Cuando utilizaron mezclas con contenidos de etanol menores, no observaron cambios significativos en las emisiones.

Zhang et al. [32] encontraron que con una tasa del $20 \%$ de EGR, las emisiones de HC aumentan entre 31 y $60 \%$ con el MCl-EP operando con gasolina, mientras que para los combustibles con etanol la tendencia en las emisiones es totalmente diferente. Se encontró que con el aumento del contenido de etanol hasta E20, las proporciones de concentración para HC están por debajo de 11,1\% con respecto a EO.

Eyidogan et al. [25] hallaron disminuciones en la temperatura de los gases de escape para E5 y E10 con el vehículo operando a $80 \mathrm{~km} / \mathrm{h}$; en promedio esta reducción es de $3{ }^{\circ} \mathrm{C}$ a 25 , 50 y $75 \%$ de carga. No obstante, a $100 \%$ de carga la mezcla E5 presenta un incremento en 
la temperatura de $2{ }^{\circ} \mathrm{C}$ respecto a la mezcla EO. Con el vehículo operando a $100 \mathrm{~km} / \mathrm{h}$ y con la mezcla E5, la temperatura de los gases de escape no presenta una diferencia respecto a cuando el vehículo opera con EO. No obstante, con el vehículo trabajando con la mezcla E10 la temperatura de los gases de escape es menor $15{ }^{\circ} \mathrm{C}$ respecto al vehículo que trabaja con la mezcla E0.

Costaglioga et al. [41], para las pruebas utilizaron un $\mathrm{MCl}$-EP de 1,6 L, de inyección, operando con E0, E10, E20, E30 y E85. Encontraron que las emisiones reguladas, cuando el $\mathrm{MCl}$ opera con las mezclas de etanol, presentan una reducción de las emisiones. La mayor reducción se asocia a E85, con la cual se obtuvo una reducción de $20 \%$ de THC y alrededor del $15 \%$ de CO y NOx. También se logró una reducción significativa del número de partículas (NP) y masa de material particulado en el rango de 1 micra (MP1), las cuales fueron de casi el $90 \%$. Para las emisiones no reguladas se logró una reducción del 50 \% de benceno y $1,3 \%$ en butadieno con la mezcla E85, el cual es clasificado como cancerígeno para los seres humanos. En cuanto a los PAHs y equivalentes tóxicos evaluados para las mezclas de alcohol y gasolina se reducen entre 30 y $70 \%$ en comparación con EO. También para esta clase de compuestos, el mejor resultado en términos de reducción de emisiones de PAHs se obtiene con la mezcla E85.

Los resultados experimentales encontrados por Hakan [36] han demostrado que las fracciones molares de CO podrían reducirse 44,26 \% para una relación de compresión de 7,75:1, y 41,67 \% para una relación de compresión de 8,25:1. Las reducciones en la emisión de CO teórico son 61,82 \% para una relación de compresión de 7,75:1, y de 68,16 \% para una relación de compresión de 8,25:1, mientras que las fracciones molares de NOx aumentan ligeramente para la mezcla de etanol con gasolina E16.5.

Chen et al. [42] probaron en un $\mathrm{MCl}-\mathrm{EP}$ monocilíndrico óptico, de $562 \mathrm{~cm}^{3}$, con una relación de compresión 11:1, de inyección directa, mezclas de gasolina etanol de EO, E10, E20, E50, E70 y E85, con dos condiciones de temperatura ambiente. Encontraron que con un incremento en la adición de etanol en la mezcla se genera un aumento tanto en el número de partículas como en la masa en el rango de tamaño entre 23 a 700 nm. Además, las relaciones entre los valores máximos y mínimos en términos de total de PN y PM son de 16 y 11, respectivamente, en condiciones de frío, así como de 7 y 8 , respectivamente, en condiciones cálidas.

He et al. [43] probaron mezclas de etanol gasolina E0 E10 y E30, en un $\mathrm{MCl}-\mathrm{EP}$ de inyección multipunto con relación de compresión de 8,2:1. Encontraron que a carga completa a 2.000 rpm, la mezcla E10 y E30 presentan una disminución de las emisiones de CO de 4,7 y 5,8 \% respectivamente, comparado con E0. Para 3.000 rpm, las emisiones de CO disminuyen en 5,7 y $3,1 \%$, respectivamente en comparación con EO. Por otro lado, las emisiones de THC con el $\mathrm{MCl}$ operando con E10 y E30 se reducen en 13,6 y $15 \%$ a 2.000 rpm, y a 3.000 rpm se reducen entre 5 y 15,3\% con respecto a E0. También se puede observar que para E10 disminuye ligeramente CO, THC y NOx, pero con la mezcla E30 las emisiones de CO, THC y NOx se reducen $35,7,53,4$ y $33 \%$, respectivamente, lo que indica que el combustible con alto contenido de oxígeno puede mejorar la combustión y, por ende, reducir las emisiones. Además, en este estudio se encontró que las emisiones de acetaldehídos se incrementan con el aumento de etanol en la mezcla. 
Al-Hasan [7] usó mezclas de gasolina sin plomo con etanol, y determinó que estas mezclas conducen a una reducción significativa de las emisiones de escape en alrededor de 46,5 y $24,3 \%$ de los valores medios medidos para CO y emisiones de $\mathrm{HC}$, respectivamente, en todo el rango de revoluciones del motor. Por otro lado, las emisiones de $\mathrm{CO}_{2}$ aumentan aproximadamente un 7,5\% causadas por un proceso de combustión máscompleto. Gravalos et al. [44] utilizaron un MCI-EP, monocilíndrico, enfriado por aire, con carburador, mezclas de etanol con gasolina en proporciones de E10, E15, E20, E25 y E30. Observaron que la emisión de HC era 838 ppm vol. a 1.600 rpm cuando el motor se alimenta con gasolina sin plomo; mientras que la emisión de HC fue 667 ppm vol. para la mezcla E25; esto indica que la emisión de HC se redujo un 20,4\%.

Graham et al. [45] usaron tres vehículos MClEP de inyección multipunto, con normas de emisión diferentes, los cuales fueron: Ford Escort ZX2 modelo 1998 (US EPA Tier 1 estándar de emisión, aproximadamente 80.000 km), Nissan Sentra modelo 2001 (California SULEV norma cero de emisiones por evaporación, aproximadamente 12.000 km) y una Dodge Caravan modelo 2003 (Norma de emisiones US EPA LEV, combustible flexible, aproximadamente $25.000 \mathrm{~km}$ ). Además, utilizaron uno de inyección directa: Mitsubishi Dion Exceed modelo 2000 (Norma de emisiones LEV japonesa, aproximadamente $25.000 \mathrm{~km})$. Probando el Escort y el Sentra a 20 y $-10{ }^{\circ} \mathrm{C}$ de temperatura ambiente y la Caravan y la Dion a $20{ }^{\circ} \mathrm{C}$, los resultados obtenidos en los vehículos al operar con E10, muestran disminuciones en las emisiones de CO de un $16 \%$, aumentos en NMHC, NMOG, acetaldehído, 1,3-butadieno y benceno en un $9 \%, 14 \%, 108 \%, 16 \%$ y $15 \%$, respectivamente, y ningún cambio estadísticamente significativo en NOx, formaldehído, emisiones de $\mathrm{CO}_{2} \mathrm{CH}_{4}$ y $\mathrm{N}_{2} \mathrm{O}$. Con E85 se encontraron disminuciones significativas en las emisiones de NOx, NMHC, 1,3-butadieno y benceno en un $45 \%, 48 \%, 77$ $\%$ y $76 \%$, respectivamente, así como aumentos significativos en las emisiones de formaldehído en un $73 \%$ y acetaldehído en un $2.540 \%$, y sin cambios estadísticamente significativos en las emisiones de $\mathrm{CO}, \mathrm{CO}_{2}$ y NMOG.

Tavares et al. [46] probaron un analizador de gases electroquímico Tempest 100 con diferentes vehículos operando con EO y E100, y encontraron que las emisiones de NOx fueron claramente inferiores en los vehículos que operaron etanol, pero para las emisiones de $\mathrm{CO}_{2}$ y $\mathrm{CO}$ esta diferencia no fue significativa. Parámetros como la velocidad, el año de fabricación, el tipo, el mantenimiento, son en algunas ocasiones las variables más importantes en el análisis de las emisiones contaminantes en un vehículo, incluso por encima del tipo de combustible con el que opera el motor.

Ghazikhani et al. [47] emplearon un MCl-EP monocilíndrico de dos tiempos, mezclas E5, E10 y E15. Las pruebas se realizaron en un dinamómetro de chasís a 4 regímenes de giro del motor $(2.500,3.000,3.500$ y 4.500 rpm) y cuatro cargas diferentes $(25,50,75$ y $100 \%$ ). Encontraron que cuando la velocidad del motor aumenta, la relación combustible/ aire disminuye, razón por la cual los HC se reducen un $30 \%$ en promedio. Cuando aumenta la velocidad del motor, el tiempo requerido para la combustión disminuye, por lo que se incrementan las emisiones de CO. En general, para diferentes velocidades y cargas, el contaminante CO disminuyó en promedio un $35 \%$. Con $\mathrm{E} 5$, el $\mathrm{CO}_{2}$ disminuyó aproximadamente $6,3 \%$. La mayor ventaja de las mezclas de etanol es la reducción de NOx, 
los cuales se reducen en $83 \%$ cuando se utiliza E15 y en $38 \%$ en promedio para otros casos.

Canakci et al. [38] utilizaron un vehículo comercial a una velocidad de $80 \mathrm{~km} / \mathrm{h}$ y 100 $\mathrm{km} / \mathrm{h}$ con las mezclas descritas anteriormente. Determinaron que las disminuciones en las emisiones de CO para E5 y E10 son 18 y $17 \%$, en promedio, comparado con EO. Igualmente, la disminución de las emisiones de CO para E10 es el $3 \%$ en comparación con EO. Las disminuciones en la emisión de $\mathrm{CO}_{2}$ para E5 y E10 son 9,5 y $8 \%$, respectivamente, en comparación con EO. Las emisiones promedio de $\mathrm{CO}_{2}$ de $\mathrm{E} 5$ y E10 disminuyeron 4 y 3,7 \% a $100 \mathrm{~km} / \mathrm{h}$, respectivamente. La disminución en las emisiones de HC para E5 y E10, en comparación con E0, fueron 27 y $32 \%$ a a 80 $\mathrm{km} / \mathrm{h}$. Las disminuciones en las emisiones de HC para E5 y E10 fueron 12 y $16 \%$, en promedio, en comparación con EO. La disminución de las emisiones de NOx de E5 y E10, en comparación con E0, fueron 11 y 15,5\% a $80 \mathrm{~km} / \mathrm{h}$. A una velocidad de $100 \mathrm{~km} / \mathrm{h}$, las emisiones de NOx de E5 y E10 disminuyeron un 10,5 y 13,5 \%, respectivamente.

Karavalakis et al. [48] evaluaron siete vehículos de diferente tecnología, dos de tecnología de catalizador de tres vías (Toyota pickup modelo 1984, Nissan Truck 1985), dos vehículos Tech 4 (Ford Explorer 1991, Ford Festiva 1993), tres con tecnología Tech 5 (Honda Accord 1996, Toyota Camry 2000, Chevrolet Silverado 2007), con el uso de mezclas de E10, E20, E50 y E85, y encontraron que para el Ford Festiva 1993, los NOx aumentan un $13,2 \%$ para E10 y $24,6 \%$ para E20. Reducciones de HC fueron de 17,4 y $22,7 \%$ para E10 y E20, respectivamente, para la Toyota pickup 1984. Reducciones de HC de un $12,2 \%$ para E10 y $23 \%$ para E20 fueron observados en el Nissan pickup 1985. Las reducciones de las emisiones de CO para
E20 fueron 72,2 \% para el Toyota 1984, 36,4\% para el Nissan 1985 y del 32,8\% para el Honda Accord 1996. Para el Honda Accord 1996 las emisiones de acetaldehído aumentaron para la mezcla E10 en un $71 \%$, mientras que E20 aumentó $202 \%$ en comparación con EO. Para Chevy Silverado 2007, aumentos significativos en acetaldehído solo se observaron con el uso del combustible E85, con incrementos del orden de $197 \%$ en comparación con EO. Las emisiones de acetaldehído para E10 fueron $39 \%$ más bajas que con EO. Las reducciones en la emisión de formaldehído fueron $44 \%$ para E10, 36\% para E20 y $27 \%$ para E50, en comparación con E0. El uso de E85 resultó en un aumento significativo en las emisiones de formaldehído con un aumento del $88 \%$ en comparación con EO. El vehículo Honda 1996 mostró reducciones en las emisiones de acetona entre 39 y $56 \%$, con niveles superiores de etanol en la mezcla. El Chevrolet Silverado 2007, presenta las reducciones más altas de acetona al operar con E10, con reducciones de $63 \%$ en comparación con E0. Además, para E10 y E20 las emisiones crotonaldehído aumentaron un 510 y $327 \%$, respectivamente, en comparación con EO.

Paulopoulos et al. [49] evaluaron E0, E3 y E10 en un vehículo Opel 1.6 L con convertidor catalítico de tres vías y encontraron que con la adición del etanol al combustible hasta un $10 \% \mathrm{v} / \mathrm{v}$, se obtuvo una disminución de las emisiones de CO en todo el rango de operación. Las emisiones de los motores, como el acetaldehído, se incrementaron de manera significativa para los combustibles con etanol, casi el doble en algunos casos. La operación del convertidor catalítico disminuye las emisiones de acetaldehído en gran medida en el caso de E10, mientras que se observó una baja eficiencia catalítica en acetaldehído para E3. Generalmente, las emisiones de 
benceno y tolueno se redujeron por la adición de etanol en la gasolina, aunque este efecto beneficioso del etanol se eliminó después de la operación del catalizador. El ácido acético se detectó en los gases de escape solo para la mezcla E1 y E3. En cuanto a la eficacia del convertidor catalítico, se observaron grandes diferencias de un compuesto a otro; acetona, benceno, tolueno y etileno se descomponen fácilmente, mientras que etanol, hexano y acetaldehído eran bastante resistentes. Como resultado de la diferente eficiencia catalítica para cada compuesto, cada uno, etileno, metano y acetaldehído, fueron los principales compuestos presentes en la salida del motor antes de la operación del catalizador; mientras que el metano, el acetaldehído y etanol fueron los principales compuestos en las emisiones para combustibles con etanol.

Lee et al. [50] evaluaron mezclas de etanol y gasolina E0, E10 y E85 en un vehículo con $\mathrm{MCl}$-EP de 4 cilindros en línea, de $2 \mathrm{~L}$, en el ciclo de manejo NEDC, y encontraron que los niveles de emisión THC se redujeron en $20 \%$ y la concentración de NOx se redujo $30 \%$, en comparación con EO. Cuando lambda es 0.8, los niveles de concentración de partículas aumentan hasta un orden de magnitud en la concentración en comparación con la relación de lambda 1,2. A medida que el tiempo de encendido se retarda, el THC en un motor que opera con E10 disminuye un $15 \%$ y las emisiones de NOx de E10 se redujeron en un máximo de $13 \%$, en comparación con EO. A medida que el tiempo de la apertura de la mariposa se retrasa, los niveles de THC se reducen en un $10 \%$ para EO y $14 \%$ para E10. Las emisiones de NOx se incrementan en $33 \%$ para E0 y $39 \%$ para E10. Retardar la apertura de la mariposa reduce las concentraciones de partículas para E0 y E10. Encontraron que la formación de partículas fue muy dependiente del rango de velocidad del vehículo. También los niveles de emisión de partículas para E85 son inferiores a los de EO y E10 a alta velocidad. Las concentraciones de partículas totales emitidas durante el NEDC son 2,14E+11 partículas $/ \mathrm{km}$ para E0, $1.53 \mathrm{E}+11$ partículas/km para el E10 y $1.35 \mathrm{E}+11$ partículas/km para el E85. Las concentraciones de partículas totales emitidas durante el NEDC para E85 se redujeron en 37\% respecto a EO.

\section{CONCLUSIONES}

Con base en los estudios analizados en el presente artículo se puede establecer que el uso de etanol mezclado con gasolina en porcentajes mayores al $20 \%$ como combustible no representa una disminución del rendimiento del motor. Además, esta adición sí genera una disminución en las emisiones de $\mathrm{CO}, \mathrm{CO}_{2}$ y $\mathrm{HC}$. No obstante, no se observa una tendencia para las variables de temperatura de gases y NOx, ya que algunos autores reportan disminuciones y otros aumentos de estas variables.

De acuerdo con los resultados obtenidos por los autores, los procesos de combustión mejoran con el uso de mezclas etanol-gasolina; este comportamiento es causado principalmente por la mayor cantidad de oxígeno disponible en la mezcla aire-combustible. Además, se encontró que el etanol genera más energía térmica dentro del cilindro durante la combustión. [51]

El aumento en la relación de compresión del MCl-EP mejora el desempeño de este, para mezclas superiores al $20 \%$ de etanol, ya que permite un mayor ingreso de aire, lo cual mejora los parámetros de combustión, y se obtiene así un aumento en el torque y la eficiencia térmica. Por otro lado, los resultados obtenidos por 
diferentes investigadores muestran que con mezclas mayores a E10 se presenta una mejor combustión, lo que conduce a una reducción significativa en las emisiones reguladas sin afectar el desempeño del motor.

Con la utilización de EGR en el motor, cuando opera con mezclas de gasolina y etanol, este presenta una disminución significativa de los HC. Adicionalmente, varios estudios han encontrado que la adición de etanol en la gasolina disminuye el contenido de compuestos aromáticos en los combustibles de mezcla. Esto se debe a que el contenido de oxígeno en los combustibles de mezcla conduce a una menor velocidad de formación de hollín y mayor tasa de oxidación de este. [52]

Las tendencias de los estudios analizados muestran gran interés en la determinación de las mezclas óptimas de gasolina y etanol, con el fin de alcanzar el mejor desempeño del vehículo, al mismo tiempo que se desea lograr una reducción significativa de las emisiones contaminantes. Se observa que estos dos parámetros presentan gran dependencia de otros factores mecánicos, como el régimen de giro del motor, la relación de compresión, la temperatura dentro de la cámara de combustión, entre otros; de forma que no es sencillo establecer los parámetros óptimos de desempeño adecuados para todos los motores que operan con determinadas mezclas de etanol gasolina.

\section{GLOSARIO}

BMEP.............. Presión media efectiva al freno BSFC.... Consumo específico de combustible al freno CAD.................. Grados de ángulo del cigüeñal $\mathrm{CH}_{4}$ Metano CO..................................... Monóxido de carbono
$\mathrm{CO}_{2}$ Dióxido de carbono

E.

EC Mezclas gasolina etanol anhídrido

EGR Encendido por compresión

EH. Recirculación de gases de escape EP. Mezclas de gasolina etanol hidratado EP.. Encendido provocado EPA............ Agencia de Protección Medioambiental IMEP. Presión media efectiva a la indicada LEV. Bajas emisiones en vehículos

LHV Poder calorífico inferior $\mathrm{MCl}$ Motor de combustión interna MP. Material particulado $\mathrm{N}_{2} \mathrm{O}$ Óxido nitroso NEDC $\mathrm{NMHC}$ NMOG NOX NP........ PAHs PMS. rpm...... SULEV. revoluciones por minuto Súper ultra bajas de emisiones en vehículos

THC US. Hidrocarburos totales sin quemar VOC Estados Unidos Compuestos orgánicos volátiles

\section{BIBLIOGRAFÍA}

[1] Agarwal, A. K. (2007). Biofuels (alcohols and biodiesel) applications as fuels for internal combustion engines. Progress in Energy and Combustion Science, 33, pp. 233-271. doi:10.1016/j. pecs.2006.08.003

[2] Sadeghinezhad, E., Kazi, S. N., Sadeghinejad, F., Badarudin, A., Mehrali, M., Sadri, R., Reza, M. (2014). A comprehensive literature review of biofuel performance in internal combustion engine and relevant costs involvement. Renewable and Sustainable Energy 
Reviews, 30, pp. 29-44. doi:10.1016/j. rser.2013.09.022

[3] García, C., Manzini, F., Islas, J. (2010). Air emissions scenarios from ethanol as a gasoline oxygenate in Mexico City Metropolitan Area. Renewable and Sustainable Energy Reviews, 14, pp. 3032-3040. doi:10.1016/j. rser.2010.07.011

[4] Manzetti, S., Andersen, O. (2015). A review of emission products from bioethanol and its blends with gasoline. Background for new guidelines for emission control. Fuel, 140, pp. 293-301. doi:10.1016/j. fuel.2014.09.101

[5] Yüksel, F., Yüksel, B. (2004). The use of ethanol-gasoline blend as a fuel in an $\mathrm{SI}$ engine. Renewable Energy, 29, pp. 1181-1191. doi:10.1016/j. renene.2003.11.012

[6] Pourkhesalian, A., Shamekhi, A., Salimi, F. (2010). Alternative fuel and gasoline in an SI engine: A comparative study of performance and emissions characteristics. Fuel, 89, pp. 1056-1063. doi:10.1016/j.fuel.2009.11.025

[7] Al-Hasan, M. (2003). Effect of ethanolunleaded gasoline blends on engine performance and exhaust emission. Energy Conversion and Management, 44, pp. 1547-1561. doi:10.1016/S01968904(02)00166-8

[8] Ozsezen, A., Canakci, M. (2011). Performance and combustion characteristics of alcoholegasoline blends at wide-open throttle. Energy, 36, pp. 2747-2752. doi:10.1016/j.energy.2011.02.014
[9] Niven, R. (2005). Ethanol in gasoline: environmental impacts and sustainability review article. Renewable and Sustainable Energy Reviews, 9, pp. 535-555. doi:10.1016/j. rser.2004.06.003

[10] Naik, S. N., Goud, V., Rout, P., Dalai, A. (2010). Production of first and second generation biofuels: A comprehensive review. Renewable and Sustainable Energy Reviews, 14, pp. 578-597. doi:10.1016/j. rser.2009.10.003

[11] Sánchez, C., Cardona O. (2007). Fuel ethanol production process design trends and integration opportunities. Bioresource Technology, 98, pp. 2415-2457. doi:10.1016/j.biortech.2007.01.002

[12] Mathewson, S. W. (1980). The Manual for the Home and Farm Production of Alcohol Fuel, Ten Speed Press.

[13] Balat, M., Balat, H. (2009). Recent trends in global production and utilization of bio-ethanol fuel. Applied Energy, 86, pp. 2273-2282. doi:10.1016/j. apenergy.2009.03.015

[14] Kumar, S., Singh, N., Prasad, R. (2010). Anhydrous ethanol: A renewable source of energy. Renewable and Sustainable Energy Reviews, 14, pp. 1830-1844. doi:10.1016/j.rser.2010.03.015

[15] Surisetty, V., Dalai, A., Kozinski, J. (2011). Alcohols as alternative fuels: An overview. Applied Catalysis A: General, 1, pp. 1-11. doi:10.1016/j.apcata.2011.07.021

[16] Gray, K., Zhao, L., Emptage, M. (2006). Bioethanol. Biocatalysis and 
biotransformation, 10, pp. 141-146. doi:10.1016/j.cbpa.2006.02.035

[17] Kumar, A., Jones, D. D., Hanna, M. A. (2009). Thermochemical biomass gasification: a review of the current status of the technology. Energies, 2(3), pp. 556-581. doi:10.3390/en20300556

[18] Winther, M., Møller, F., Jensen, T. (2012). Emission consequences of introducing bio ethanol as a fuel for gasoline cars. Atmospheric Environment, 55, pp. 144-153. doi:10.1016/j. atmosenv.2012.03.045

[19] Fedebiocombustibles (2010). En: http:// www.fedebiocombustibles.com/notaweb-id-923.htm.

[20] Departamento Nacional de Planeación (2008). Lineamientos de politica para promover la produccion sostenible de biocombustibles en Colombia, Conpes 3510. Documento Conpes 3510, Bogotá.

[21] Costa, R., Sodré, J.(2011). Compressionratio effects on an ethanol/gasoline fuelled engine performance. Applied Thermal Engineering, 31, pp. 278-283. doi:10.1016/j. applthermaleng.2010.09.007

[22] Yücesu, H., Topgül, T., Cinar, C., Okur, M. (2006). Effect of ethanol-gasoline blends on engine performance and exhaust emissions in different compression ratios. Applied Thermal Engineering, 26, pp. 2272-2278. doi:10.1016/j. applthermaleng.2006.03.006

[23] Hsieh, W., Chen, R., Wu, T., Lin, T. (2002). Engine performance and pollutant emission of an SI engine using ethanol- gasoline blended fuels. Atmospheric Environment, 36, pp. 403-410. doi:10.1016/S1352-2310(01)00508-8

[24] Celik, M. (2008). Experimental determination of suitable ethanol-gasoline blend rate at high compression ratio for gasoline engine. Applied Thermal Engineering, 28, pp. 396-404. doi:10.1016/j. applthermaleng.2007.10.028

[25] Eyidogan, M., Ozsezen, A., Canakci, M., Turkcan, A. (2010). Impact of alcoholgasoline fuel blends on the performance and combustion characteristics of an SI engine. Fuel, 89, pp. 2713-2720. doi:10.1016/j.fuel.2010.01.032

[26] Costa, R., Sodré, J. (2010). Hydrous ethanol vs. gasoline-ethanol blend: Engine performance and emissions. Fuel, 89, pp. 287-293. doi:10.1016/j. fuel.2009.06.017

[27] Park, Ch., Choi, Y., Kim, Ch., Oh, S., Lim, G., Moriyoshi, Y. (2010). Performance and exhaust emission characteristics of a spark ignition engine using ethanol and ethanol-reformed gas. Fuel, pp. 21182125. doi:10.1016/j.fuel.2010.03.018

[28] Zhuang, Y., Hong, G. (2013). Primary investigation to leveraging effect of using ethanol fuel on reducing gasoline fuel consumption. Fuel, pp. 425-431. doi:10.1016/j.fuel.2012.09.013

[29] Balki, M., Sayin, C., Canakci, M. (2014). The effect of different alcohol fuels on the performance, emission and combustion characteristics of a gasoline engine. Fuel, 115, pp. 901-906. doi:10.1016/j. fuel.2012.09.020 
[30] Topgül, T., Yücesu, H., Cinar, C., Koca A. (2006). The effects of ethanol-unleaded gasoline blends and ignition timing on engine performance and exhaust emissions. Renewable Energy, 31, pp. 25342542. doi:10.1016/j.renene.2006.01.004

[31] Wu, Ch., Chen, R., Pu, J., Lin, T. (2004). The influence of air-fuel ratio on engine performance and pollutant emission of an SI engine using ethanol-gasolineblended fuels. Atmospheric Environment, 38, pp. 7093-7100. doi:10.1016/j. atmosenv.2004.01.058

[32] Zhang, Z., Wang, T., Jia, M., Wei, Q., Meng, X., Shu, G. (2014). Combustion and particle number emissions of a direct injection spark ignition engine operating on ethanol/gasoline and n-butanol/gasoline blends with exhaust gas recirculation. Fuel, 130, pp. 177-188. doi:10.1016/j. fuel.2014.04.052

[33] Schifter, I., Díaz, L., Gómez, J. P., González, U. (2013). Combustion characterization in a single cylinder engine with mid-levels hydrated ethanol-gasoline blended fuels. Fuel, 103, pp. 292-298. doi:10.1016/j. fuel.2012.06.002

[34] Turner, D., Xu, H., Cracknell, R., Natarajan, V., Chen, X. (2011). Combustion performance of bio-ethanol at various blend ratios in a gasoline direct injection engine. Fuel, 90, pp. 1999-2006. doi:10.1016/j.fuel.2010.12.025

[35] Wua, X., Daniel, R., Tian, G., Xu, H., Huang, Z., Richardson, D. (2011). Dual-injection: The flexible, bi-fuel concept for sparkignition engines fuelled with various gasoline and biofuel blends. Applied
Energy, 88, pp. 2305-2314. doi:10.1016/j. apenergy.2011.01.025

[36] Hakan, B. (2005). Experimental and theoretical investigation of using gasolineethanol blends in spark-ignition engines. Renewable Energy, 30, pp. 1733-1747. doi:10.1016/j.renene.2005.01.006

[37] Kumar, J., Trivedi, D., Mahara, P., Butola, R. (2013). Performance Study of Ethanol Blended Gasoline Fuel in Spark Ignition Engine. Journal of Mechanical and Civil Engineering, 7(3), pp. 71-78.

[38] Canakci, M., Ozsezen, A., Alptekin, E., Eyidogan, M. (2013). Impact of alcoholegasoline fuel blends on the exhaust emission of an $\mathrm{SI}$ engine. Renewable Energy, 52, pp. 111-117. doi:10.1016/j.renene.2012.09.062

[39] Heywood, J. (1988). Internal Combustion Engines Fundamentals, McGraw-Hill, pp. 497.

[40] Schifter, I., Díaz, L., Rodríguez, R., Gómez, J. P., González, U. (2011). Combustion and emissions behavior for ethanol-gasoline blends in a single cylinder engine. Fuel, 90, pp. 3586-3592. doi:10.1016/j. fuel.2011.01.034

[41] Costagliola, M. A., De Simio, L., lannaccone, S., Prati, M. V. (2013). Combustion efficiency and engine out emissions of a S.I. engine fueled with alcohol/gasoline blends. Applied Energy, 111, pp. 1162-1171. doi:10.1016/j. apenergy.2012.09.042

[42] Chen, L., Stone, R., Richardson, D. (2012). A study of mixture preparation and PM 
emissions using a direct injection engine fuelled with stoichiometric gasoline/ ethanol blends. Fuel, 96, pp. 120-130. doi:10.1016/j.fuel.2011.12.070

[43] He, B., Wang, J., Hao, J., Yan, X., Xiao, J. (2003).Astudyonemissioncharacteristics of an EFI engine with ethanol blended gasoline fuels. Atmospheric Environment, 37, pp. 949-957. doi:10.1016/S13522310(02)00973-1

[44] Gravalos, I., Moshou, D., Gialamas, T., Xyradakis, P., Kateris, D., Tsiropoulos, Z. (2013). Emissions characteristics of spark ignition engine operating on lowerehigher molecular mass alcohol blended gasoline fuels. Renewable Energy, 50, pp. 27-32. doi:10.1016/j.renene.2012.06.033

[45] Graham, L., Belisle, S., Baas, C. (2008). Emissions from light duty gasoline vehicles operating on low blend ethanol gasoline and E85. Atmospheric Environment, 42, pp. 4498-4516. doi:10.1016/j.atmosenv.2008.01.061

[46] Tavares, R., Sthel, M. S., Campos, L. S., Rocha, M. V., Lima, G. R., da Silva, M. G., Vargas H. (2011). Evaluation of Pollutant Gases Emitted by Ethanol and Gasoline Powered Vehicles. Procedia Environmental Sciences, 4, pp. 51-60. doi:10.1016/j. proenv.2011.03.007

[47] Ghazikhani, M., Hatami, M., Safari, B., Ganji, D. (2014). Experimental investigation of exhaust temperature and delivery ratio effect on emissions and performance of agasoline-ethanol twostroke engine. Case Studies in Thermal Engineering, 2, pp. 82-90. doi:10.1016/j. csite.2014.01.001
[48] Karavalakis, G., Durbin, T., Shrivastava, M., Zheng, Z., Villela, M., Jung, $H$. (2012). Impacts of ethanol fuel level on emissions of regulated and unregulated pollutants from a fleet of gasoline lightduty vehicles. Fuel, 93, pp. 549-558. doi:10.1016/j.fuel.2011.09.021

[49] Poulopoulos, S. G., Samaras, D. P., Philippopoulos, C. J. (2001). Regulated and unregulated emissions from an internal combustion engine operating on ethanol-containing fuels. Atmospheric Environment, 35, pp. 4399-4406. doi:10.1016/S1352-2310(01)00248-5

[50] Lee, H., Myung, Ch., Park, S. (2009). Time-resolved particle emission and size distribution characteristics during dynamic engine operation conditions with ethanol-blended fuels. Fuel, 88, pp. 16801686. doi:10.1016/j.fuel.2009.03.007

[51] Turns, S. R. (2000). An introduction to combustion: Concepts and applications, Mc Graw Hill.

[52] Esarte, C., Abián, M., Millera, Á., Bilba, R., Alzueta, M. U. (2012). Gas and soot products formed in the pyrolysis of acetylene mixed with methanol, ethanol, isopropanol or n-butanol. Energy, 43, pp. 37-46. doi:10.1016/j. energy.2011.11.027 